Eine ebenso nüchterne, aber stärker an liberaler Institutionenentwicklung orientierte Sichtweise verfolgt Luka Biong Deng (Biong Deng und Logan, 2019: 262-274). Abraham Awolich (2015) wiederum argumentiert in Richtung einer abgerissenen Entwicklung einer nationalen Identität, nicht zuletzt hervorgerufen durch ein Leadership-Problem der staatlichen Eliten.

Andere Beiträge arbeiten die Geschichte des Widerstandes aus zumeist persönlicher Perspektive auf (vgl. etwa Fuli Boki Tombe Ga'le, 2002; MadutArop, 2006). Eine zentrale Rolle in diesen Arbeiten kommt dem Nasir-Split innerhalb der SPLM/A im Jahr 1991 zu (vgl. etwa Nyaba, 2000, 2019; Akol, 2003), was nicht nur die traumatische Qualität dieses Spaltungsprozesses verdeutlicht, sondern einen Hinweis darauf gibt, dass die dafür maßgebliche Fraktionalisierung ein bis heute manifester, institutionalisierter Wesenszug der SPLM/A ist.

Das wohl interessanteste, wenn auch nicht einfach zugängliche Werk aus südsudanesischer Perspektive hat Kuyok Abol Kuyok (2015) vorgelegt. Kuyok Abol hat in differenzierter Weise und nach sicherlich mühsamer Recherche auf weit über tausend Druckseiten die Biografien aller namhaften Exponent:innen der südsudanesischen Geschichte zusammengetragen. Gerade seine Darstellungen erinnern daran, dass politische Kontroversen, selbst wenn ihre Austragung in eine bewaffnete Form kippt, nicht einfach als "mangelnde Reife« abgekanzelt werden können. Südsudanesische Politik ist seit ihrer Entstehung ein ernsthaftes Geschäft, das mit hohem Einsatz und einer einzigartigen Palette an Fähigkeiten und Fertigkeiten gespielt wird. $\mathrm{Zu}$ viele haben zu viel investiert und zu viel geopfert, um das Land und seine Geschichte auf die leichte Schulter zu nehmen.

\title{
Prästaatliche Institutionalisierungen
}

Jede Auseinandersetzung mit südsudanesischer Geschichte muss zunächst einmal die geografischen Dimensionen hervorheben, die dieses Land auszeichnen. Der historische Sudan ist besser als Subkontinent denn als werdender Nationalstaat zu verstehen. Vor seiner Spaltung war der Sudan das größte Land Afrikas. In seiner einstmaligen Ausdehnung nach Westen, die Teile des heutigen Tschad und der Zentralafrikanischen Republik umfasste, war er tatsächlich von monumentalem Ausmaß. Zugleich ging diese Größe aufgrund der herausfordernden klimatischen Bedingungen nie mit einer hohen Bevölkerungszahl einher. Selbst im historischen Sudan war die Bevölkerungsdichte 
außerhalb des Ballungszentrums Khartum und einiger Teile Darfurs gering. Der südliche Sudan war Zeit seiner Geschichte nur dünn besiedelt.

Dies hat sich nach der staatlichen Unabhängigkeit nicht geändert. Wenngleich mit dem historischen Sudan nicht vergleichbar ist der Südsudan immer noch eines der territorial gemessen größeren Länder der Erde. Mit etwa $644.000 \mathrm{~km}^{2}$ ist seine Fläche mit Frankreich, Afghanistan oder Texas vergleichbar. Die Einwohner:innen-Zahl ist, so schwer sie festzustellen ist, demgegenüber verschwindet gering. Der letzte Zensus liegt über zehn Jahre zurück, und annähernd zwei Millionen Südsudanes:innen leben als Schutzsuchende außerhalb der Staatsgrenzen, was jede Schätzung erschwert. Nach Prognosen des UN World Population Prospect bewegt sich der Südsudan 2020 bei etwa 11,2 Millionen Einwohner:innen, was 17,5 Einwohner:innen pro $\mathrm{km}^{2}$ entsprechen würde. Dies ist für einen Flächenstaat, der weder durch große Wüstengebiete noch durch Permafrost-Zonen gekennzeichnet ist, ein bemerkenswert niedriger Wert, selbst wenn die Wirkung des Sudd - ein großes Sumpfgebiet im Oberlauf des Weißen Nil, jenem Fluss, der das Land von Uganda kommend durchzieht - in Betracht gezogen wird.

Umweltfaktoren haben einen wesentlichen Einfluss auf die institutionelle Entwicklung der Region (Beswick, 2004: 9). Diese Bedingungen stehen einer einheitlichen Form von Durchstaatlichung strukturell entgegen. So entstanden gemeinschaftliche Institutionen, die zumindest einen relevanten Teil des heutigen Staatsgebietes erfassen, nur langsam. Solche Formen der Institutionalisierung, zumeist Handelsnetzwerke zwischen unterschiedlichen Gemeinschaften, entwickelten sich als ein Produkt von Migrationsbewegungen, die die von nomadischem Pastoralismus dominierte Region seit Jahrhunderten kennzeichnen. Diese kontinuierlichen Migrationsbewegungen und das herausfordernde Terrain, das bis ins 19. Jahrhundert die Expansion uniformer Mächte in das Gebiet effektiv verhindert hat, unterbanden die Entwicklung vereinheitlichender institutioneller Prozesse, wie sie sich etwa in weiten Teilen Asiens oder Europas über Jahrhunderte vollzogen. Die Ausdifferenzierung einer bemerkenswerten sozialen Diversität war die Folge. Nach offizieller Zählung umfasst der heutige Südsudan 64 ethnopolitische Gruppen mit ebenso vielen unterschiedlichen Sprachen. Neben den überregionalen arabischen Dialekten dominieren die eng verwandten nilotischen Sprachen Dinka und Nuer, sowie Bari und Zande. Alle anderen Sprachen werden von jeweils weniger als 200.000 Menschen gesprochen. ${ }^{1}$ 
Dinka-Gemeinschaften besiedelten die Region nach derzeitigem Kenntnisstand im 14. und 15. Jahrhundert von Norden kommend. Eine oft erzählte Geschichte, bestätigt von Stephanie Beswick (2004: 17), berichtet, dass der Name der sudanesischen Hauptstadt Khartum, circa 400 Kilometer nördlich von Renk County, dem nördlichsten Teil des heutigen Südsudan gelegen, auf einen alten Dinka-Begriff zurückgeht, der einen Ort bezeichnet, an dem sich zwei Flüsse - im Falle Khartums der Weiße und der Blaue Nil - treffen. Die Dinka-Migration traf auf einigen Widerstand, hauptsächlich von im heutigen Nubien präsenten Funj, deren 1504 gegründetes Sultanat sich bis ins heutige Eritrea erstreckte. Die nach Klans und Sub-Klans unterteilte soziale und politische Organisation der Dinka förderte die Zerstreuung ihrer Besiedlung. Padang Dinka emigrierten ab dem 17. Jahrhundert in die Regionen am Oberlauf des Weißen Nil im Einflussbereich des Shilluk-Königreiches, des einheitlichsten politischen Gemeinwesens in der Region, das sich Mitte des 16. Jahrhunderts um die Hauptstadt Fashoda, dem heutigen Kodok in Upper Nile State, formierte. Die Auseinandersetzungen waren hart und wurden zeitweise bewaffnet ausgetragen (Beswick, 2004: 34). Sie wurden aber in weiterer Folge auch von jahrzehntelangen Phasen des friedlichen Zusammenlebens abgelöst (Craze, 2019: 18).

Neben den Ngok Dinka, die sich im Bereich des heutigen Abyei ansiedelten, migrierten andere Dinka-Klans vom 16. bis ins 18. Jahrhundert in weite Teile von Greater Bahr el-Ghazal im Westen des heutigen Südsudan sowie von Jonglei im Osten. Die Dinka von Bahr el-Ghazal stellten nicht nur die klare Bevölkerungsmehrheit in der Region. Sie entwickelten sich über die folgenden Jahrzehnte zu einem der sozialen Epizentren des Widerstandes gegen den Norden. Bis zum heutigen Tag sind viele Schlüsselpositionen in SPLM und SPLA/SSPDF mit Bahr el-Ghazal Dinka besetzt. Auch Präsident Salva Kiir entstammt einer Dinka-Gemeinschaft aus Gogrial im heutigen Warrap State in Greater Bahr el-Ghazal.

In Jonglei kommt es zu einem Aufeinandertreffen der migrierenden Dinka-Gemeinschaften mit den nicht der nilotischen Sprachgruppe angehörigen Murle, die die Region als Nomaden von Äthiopien her besiedeln. Diese Berührungen nehmen unterschiedliche Formen an. Manchmal ist es ein tolerierendes Nebeneinander, mitunter eskalieren Konflikte in Gewaltanwendung. Jon Arensen, der die Murle über lange Jahre ethnografisch erforschte, verweist wiederholt auf die Kongruenz der Begriffe »Fremder« und Feind « in der Murle-Sprache. Dies wird von benachbarten Stämmen insofern aufgegriffen, als diese Murle ebenfalls schlicht als »Feinde« titulieren 
(Arensen, 1964: 1). Dennoch: Trotz mitunter martialischer Selbstdarstellungen verlief ein Großteil der Migrationsbewegungen ohne organisierte Gewaltanwendung.

Nach wie vor ungeklärt ist die historische Beziehung der Dinka zu der zweitstärksten Bevölkerungsgruppe im heutigen Südsudan, den Nuer. Die wenigen anthropologischen Arbeiten vermuten entweder, dass die Nuer von den Luo im Südosten oder, was mittlerweile als wenig wahrscheinlich gilt, sich in der oralen Geschichte aber erhalten hat, von den Dinka selbst abstammen. Auch hier dominiert die Erzählung eines lange andauernden, fast schon zur Tradition gewordenen Gewaltkonfliktes: „Dinka oral histories recount that they have been at war with the Nuer for at least three centuries.« (Beswick, 2004: 168)

Dem rezenten Kenntnisstand nach ist diese Wahrnehmung eine massive Übertreibung. Allerdings war das Verhältnis von Dinka und Nuer von jeher von besonderer Qualität, was nicht zuletzt durch die relative Größe der beiden Volksgruppen bedingt ist. Von 1820 bis 1860 kommt es zu einer substanziellen Nuer-Migrationswelle von ihrem Zentrum im heutigen Unity State nach Osten und Süden, bis nach Jonglei (Kelly, 1985). Die Ursachen dieser Migrationswelle sind nicht umfassend geklärt. Sie reichen von klimatischen Bedingungen und einem damit zusammenhängenden Bevölkerungsdruck bis hin zu den beginnenden Menschenjagden von arabischen Reiterstämmen im Norden. Dabei kam es zum Eindringen in Dinka-Siedlungsgebiete, die sich allerdings nur selten in Eroberungen, sondern graduell in einem über Jahre dauernden Prozess vollzogen. Im Zuge dessen wurden Dinka in NuerGemeinschaften assimiliert, nachdem zuvor zumeist Dinka-Gemeinschaften kleinere Stammes- und Sprachgruppen integriert hatten.

Strukturell ähneln sich die Migrationsprozesse der Dinka und der Nuer, da sie, wenn auch in unterschiedlicher Dimension, zentrifugale Expansionsbewegungen erlebten, die selbst ihre Sprache diversifizierten. Beswick (2004: 176) führt dies im Falle der Dinka auf »increasing populations, land shortages, ecological stress, and the Dinka philosophy of political atomization « zurück. Heute zählen ethnografische Untersuchungen 26 Dinka-Hauptgruppen, die selbst wiederum in zahlreiche Sub-Klans zerfallen. Wenngleich sich DinkaSprechende nach wie vor weitgehend verstehen, hat sich die Sprache, vor allem zwischen Ngok Dinka im Norden, Bor Dinka im Osten und den Bahr el-Ghazal Dinka im Westen, in distinkte Dialekte ausdifferenziert.

Diese Ausdifferenzierung von Dinka und Nuer hat politisch-institutionelle Folgen, die Beswick (ebd.: 176) prägnant als »fission politics« zusammen- 
fasst. Dieses Muster der kontinuierlichen Folge von Spaltungen beruht auf dem Fehlen übergeordneter Instanzen, die sich auf entpersonalisierte Formen von Legitimität und Autorität berufen können. Abgesehen vom ShillukKönigreich und den zeitweise strikt organisierten Zande Chiefdoms hat keine Bevölkerungsgruppe im Südsudan derartig komplexe hierarchische Organisationsformen entwickelt. Dies ist allerdings keineswegs als eine Tendenz zum Egalitarismus misszuverstehen. Soziale Unterschiede zwischen Klans und Familien können beträchtlich sein. Dennoch sind Entscheidungshierarchien in den meisten Gemeinschaften flach. In einigen Fällen gestalten sie sich phasenweise auch anarchisch, etwa bei den Murle, wenn die traditionelle Autorität von Chiefs durch sich gewaltsam entladende Spannungen zwischen organisierten Altersgruppen - den Age-Sets - unterminiert wird (vgl. Felix da Costa, 2018: 13-20).

Dieser Kurzabriss über die vorkoloniale Geschichte, die sich auf dem Gebiet des heutigen Südsudan vollzogen hat, beinhaltet zwei wesentliche Implikationen. Erstens veranschaulicht diese Geschichte das Problem der nie stattgefundenen durchschlagenden vereinheitlichenden Institutionalisierung. Dies gilt nicht nur zwischen den, sondern auch innerhalb der Bevölkerungsgruppen. Bis in die Mitte des 19. Jahrhunderts hatte sich in der Region keine Verkehrssprache durchgesetzt, die von einer Mehrzahl der Menschen verstanden oder gar gesprochen worden wäre. Einige Sprachgruppen werden assimiliert und verschwinden. Andere, wie Dinka, verbreiten sich und diversifizieren sich in unterschiedliche Dialekte. Der hohe Grad an Spezifität der institutionellen Entwicklung wird es für kommende Bemühungen um Vereinheitlichung der Kolonialmächte und des späteren unabhängigen Sudan praktisch verunmöglichen, eine erfolgreiche Durchstaatlichung durchzusetzen. Die Voraussetzungen zur Tönniesschen Transformation von Gemeinschaft zu Gesellschaft (Fukuyama, 2015: 41), wie etwa die Entwicklung ökonomischer Zentren, einer gemeinschaftlich genutzten Infrastruktur und daran anschließender kollektiver Ideale und Vorstellungen, waren nicht gegeben. Gemeinschaften waren es, die die sich entfaltenden Konfliktlandschaften trugen und bis zum heutigen Tag tragen.

Zweitens widerspricht dieser Kurzabriss einem Mythos, den auch andere rezente Forschungsbeiträge, die im Gegensatz zur kolonialen Anthropologie ihre eigene Rolle und ihren Bezugsrahmen kritisch zu reflektieren in der Lage sind, überzeugend demontieren. Allzu oft wurde die Geschichte der Migrationsbewegungen in Ost- und Zentralafrika als eine gewalttätige erzählt, die auf beständigen bewaffneten Kampagnen und Vertreibungen aufgebaut 
gewesen wäre. Selbstverständlich ist jede Art von Verallgemeinerung schwierig, umso mehr, wenn es um die historischen Gegebenheiten in mehreren Dutzend Gemeinschaften geht, die sich über hunderte Jahre ziehen. Natürlich gab es immer wieder Episoden von gewaltsamen Auseinandersetzungen zwischen und innerhalb von Gemeinschaften, Stämmen und Familien. Dies unterscheidet die Region jedoch nicht von anderen Regionen der Welt - die europäische Geschichte in diesem Zeitraum ist jedenfalls unvergleichlich blutiger.

Zugleich zeigt ein genaueres Studium der diversen Verhältnisse der Bevölkerungsgruppen untereinander ein differenziertes Bild. Migration vollzieht sich als ein gradueller Prozess des friedlichen Einsiedelns und pragmatischen Nebeneinanders, der auch Assimilation als langfristige Normalität mit sich bringen kann. Wie in den meisten anderen Weltgegenden ist eine kriegerische Tradition nicht zu leugnen. Die gewaltsamen Auseinandersetzungen beschränken sich aber zumeist auf Episoden, die selten gesamte Gemeinschaften umfassen. Identitätskonstruktionen spielen in diesem Kontext nur eine sekundäre Rolle. Die Konflikte gestalten sich zumeist pragmatisch, entlang von geografischen Gegebenheiten, Weideland, saisonalen Migrationsrouten oder Siedlungsgebieten. Wenngleich sich die Auseinandersetzungen dieser Epoche ohne unterschiedliche Identitäten nicht erklären lassen, sind es keine Identitätskonflikte. Diese Dimension entwickelt sich erst in den kommenden Jahrzehnten mit der zielgerichteten und systematischen kolonialen Durchdringung der Region.

\section{Periphere Integration}

Das 18. und frühere 19. Jahrhundert sehen das erste Mal Ambitionen der systematischen militärischen Eroberung und der Integration des Gebietes des heutigen Südsudan in größere ökonomisch-politische Systeme. Diese Vorstöße, zunächst das vom ottomanischen Ägypten durchgesetzte Turkiyya-Regime und später die Kolonialepoche unter dem britisch-ägyptischen Kondominium, versuchten, den südlichen Sudan als eine strukturelle Peripherie in ihre Herrschaftssysteme einzugliedern. Doch die angestrebte periphere Integration bleibt unvollständig und abgerissen. Sowohl Turko-Ägypten als auch die britische Kolonialmacht scheitern an einer systematischen Integration der Region. 